\title{
Microquimerismos en la enfermedad de Alzheimer y la demencia frontotemporal
}

\author{
Microchimerism in Alzheimer's disease \\ and frontotemporal dementia
}

Sara Sierra-Peláez MSc', Carlos A. Villegas-Lanau PhD²

\begin{abstract}
Resumen: las enfermedades neurodegenerativas constituyen un problema global de salud pública que tiende a incrementarse. En la búsqueda de alternativas terapéuticas frente a estas afecciones aparecen los microquimerismos fetomaternales (células fetales en estados inmaduros de diferenciación adquiridas durante el embarazo) que, al tratarse de células madre, suponen una opción terapéutica similar a la terapia celular. Estudios recientes han demostrado el papel protector de los microquimerismos en la enfermedad de Alzheimer y la esclerosis múltiple; sin embargo, al tratarse de células provenientes de otro organismo surge la posibilidad de que induzcan una respuesta inmune que lleve a la aparición o potenciación de los procesos inflamatorios propios de las enfermedades neurodegenerativas. De acuerdo con lo anterior, diversos estudios han sugerido un papel de los microquimerismos en el desarrollo de enfermedades autoinmunes. El esclarecimiento de las características, funciones, vías de ingreso, localización e interacciones de los microquimerismos con el sistema inmune del organismo hospedero es relevante en la medida en que pueden guiar el desarrollo de tratamientos frente a este tipo de enfermedades. En esta revisión se describen algunos de estos aspectos relevantes.
\end{abstract}

Palabras clave: enfermedades neurodegenerativas, enfermedad de Alzheimer, demencia frontotemporal, microquimerismo, complejo mayor de histocompatibilidad.

Abstract: Neurodegenerative diseases represent a major and increasing public health problem around the world. In the search for therapeutic alternatives against these conditions, feto-maternal microchimerisms (immature fetal cells acquired during pregnancy) appear as a possible therapeutic option similar to stem cell therapy. In fact, several studies have found that the microchimerisms play a protective role against Alzheimer's disease and multiple sclerosis. Nonetheless, given the fact that the microchimerisms are "foreign cells», they could induce an immune response, leading to the

'Sicóloga, estudiante de maestría en Ciencias Básicas Biomédicas, Universidad de Antioquia. Laboratorio de Genética Molecular. Medellín, Colombia. Correspondencia: Calle 67 \#53-108. Laboratorio 430, ciudad universitaria, Universidad de Antioquia. Correo electrónico: sarasierra86@gmail.com

2Médico, MSc en Ciencias Básicas Biomédicas, MSc en Neurociencias, PhD en Ciencias. Grupo de Neurociencias de la Universidad de Antioquia. Medellín, Colombia.

Conflicto de intereses: los autores declaran que no tienen conflicto de intereses

Medicina \& Laboratorio 2015; 21 : 333-348

Módulo 3 (Genética), número 7. Editora Médica Colombiana S.A. $2015^{\odot}$

Recibido el 16 de julio de 2015; aceptado el 16 de agosto de 2015

Medicina \& Laboratorio Volumen 21, Números 7-8, 2015. 
apparition or exacerbation of the inflammatory responses characteristic of neurodegenerative diseases. According to this, several studies have suggested a role for microchimerisms in the development of autoimmune diseases. The elucidation of characteristics, functions, ways of acquisition, paths of entry, localization, and interactions between microchimerisms and the immune system of the host can serve as a guide towards the development of a treatment against these diseases. In this review, some of these important aspects are described.

Key Words: neurodegenerative disorders, Alzheimer's disease, frontotemporal dementia, microchimerism, major histocompatibility complex.

Sierra-Peláez S, Villegas-Lanau CA. Microquimerismos en la enfermedad de Alzheimer y la demencia frontotemporal. Medicina \& Laboratorio 201 5; 21 : 333-348.

$\mathrm{L}^{2}$ as enfermedades neurodegenerativas son un amplio grupo de afecciones caracterizadas por la progresiva pérdida y degeneración neuronal y de las células de glía, cuya consecuencia clínica es con frecuencia un cuadro demencial. Este grupo de enfermedades abarcan una gran diversidad de condiciones patológicas en las que se observan cambios deletéreos en la memoria, el comportamiento y el pensamiento complejo [I].

La enfermedad de Alzheimer se caracteriza por presentar un cuadro degenerativo con pérdida progresiva de la función cognitiva y motora que en última instancia lleva a la muerte [2]. Actualmente, sólo en los Estados Unidos se registran 5.200.000 personas con Alzheimer y se espera que para el año 2050 ascienda a 13.600 .000 [3]. A nivel global se pronostica que para ese año I 5.000 .000 de personas padecerán la enfermedad [4]. La incidencia anual de la enfermedad de Alzheimer aumenta dramáticamente con la edad, pasando de 53 por cada 1.000 personas entre los 65 y 74 años, a 170 por cada 1.000 personas entre los 75 y 84 años, a 231 por cada 1.000 a partir de los 85 años, resaltando que el mayor factor de riesgo de la enfermedad de Alzheimer es el envejecimiento [3-5].

Por su parte, la demencia frontotemporal lobular se presenta más temprano en la vida por lo que es conocida como una demencia juvenil; sin embargo, alrededor del $25 \%$ de los casos aparecen en la vejez. Esta enfermedad afecta principalmente el comportamiento, el lenguaje y el estado de ánimo. Aunque su prevalencia es alrededor de 15 a 22 casos por cada 100.000 personas y su incidencia es de 2,7 a 4, I casos por cada 100.000 personas en los Estados Unidos, representa la tercera causa de demencia después del Alzheimer y la demencia por cuerpos de Lewy, y la segunda causa en las personas menores de 65 años [6]. La expectativa de vida de las personas que sufren esta enfermedad es pobre y comparable a la de la enfermedad de Alzheimer, que se relaciona con la carencia de un tratamiento efectivo frente a la misma [2,7].

Dentro de la búsqueda de tratamientos contra las enfermedades neurodegenerativas ha llamado la atención el microquimerismo fetomaternal, correspondiente a la presencia a largo plazo en el organismo de la madre de una baja cantidad de células derivadas de sus hijos [8-10]. Este microquimerismo fue hallado como un agente protector contra la enfermedad de Alzheimer en el único estudio que se ha realizado en humanos, en el cual se amplificó a partir del cerebro de mujeres muertas con o sin Alzheimer una secuencia específica del gen del cromosoma $Y$ para la detección del ADN de células masculinas, que se asume que fueron adquiridas durante 
la gestación de hijos hombres. En dicho estudio se encontró que la prevalencia y la concentración de microquimerismos masculinos de origen fetal en los cerebros de las mujeres sanas es mayor frente a las que sufrían la enfermedad [I I].

Así mismo, el microquimerismo fetomaternal adquiere relevancia en la medida en que se ha planteado como alternativa terapéutica la utilización de la terapia celular con células madre, la cual se fundamenta en la implantación selectiva de células prediferenciadas in vitro hacia diferentes etapas de maduración que, al proliferar y especializarse, reemplazan a las células con función defectuosa y restauran la actividad $[12,13]$.

A pesar de estas características, la terapia celular en degeneración neuronal aún no es una realidad, ya que sus principales hallazgos se han obtenido únicamente en modelos múridos [ 14- | 6], lo que supone un largo camino por recorrer. Dado que los microquimerismos corresponden al traspaso natural de células de un individuo a otro, se ha generado la inquietud de si pueden inducir un tipo de terapia celular o, por el contrario, generar una amplificación de la respuesta inmune y sus consecuentes procesos inflamatorios [9, I0]. De igual manera, existe la posibilidad de que los microquimerismos no tengan un efecto significativo sobre la salud y la enfermedad. Lo cierto es que actualmente no hay tratamientos disponibles efectivos ni métodos preventivos frente a la enfermedad de Alzheimer y la demencia fronto-temporal, las cuales representan un problema de salud pública a nivel global que tiende a aumentar en los próximos años.

\section{Mecanismos de adquisición de microquimerismos}

El microquimerismo se puede generar de diversas formas, mediante transfusiones de sangre, trasplante de órganos, lactancia y transferencia de células en gemelos dicigóticos por anastomosis de los vasos sanguíneos de placentas fusionadas [10]; no obstante, la forma natural de adquisición de microquimerismos se produce durante la gestación, en la que se da una transferencia por doble vía de células hematopoyéticas entre la madre y el feto a través de la barrera placentaria $[9,10]$.

El microquimerismo es un evento bastante común. Se estima que entre el $22 \%$ y el $75 \%$ de las mujeres que han tenido hijos tienen sus células en la sangre y aproximadamente el $63 \%$ las conservan décadas posteriores al parto. Por su parte, entre el $7 \%$ y el $10 \%$ de las mujeres que no han tenido embarazos poseen microquimerismos, los cuales se pueden atribuir a la transferencia de células maternas durante la gestación o de células de sus hermanos mayores que estaban presentes en la sangre de la madre o a través de contacto sexual. Los microquimerismos se han identificado en aproximadamente el $25 \%$ de las mujeres que han tenido abortos espontáneos y en el $50 \%$ de las que han tenido abortos inducidos [10,1 I ]. Sin embargo, la cantidad de microquimerismos existentes en el cerebro es bastante baja, alrededor de 0,5 gEq/ I $0^{5}$ (número de genomas quiméricos completos por cada $10^{5}$ genomas completos del huésped) [ $\mathrm{I}$ l].

\section{Microquimerismos como células madre}

Las células quiméricas tienen características propias de este tipo de células, tales como la capacidad de división y diferenciación [17]. Estudios en humanos han demostrado la transferencia bilateral de células madre hematopoyéticas, así como de células endoteliales, hepatocitos, mio- 
citos cardiacos y neuronas, entre otros $[9,10,18,19]$. El microquimerismo de origen fetal ha sido observado a partir de la sexta semana de gestación, por lo que el traspaso de células desde el embrión a la madre se puede dar en la forma de células pluripotenciales, lo que concuerda con su capacidad sospechada de diferenciación hacia cualquier tejido [18]. Entretanto, en el microquimerismo de origen materno se puede dar un traspaso de células madre multipotenciales, dando lugar a varios tipos de células de un mismo tejido, así como al traspaso de células maduras $[10,18]$.

Se sospecha que el microquimerismo tiene las mismas características de las células madre extraídas del cordón umbilical y la placenta en el momento del nacimiento, las cuales son pluripotenciales, no tumorigénicas y no generan respuesta inmune dado que no presentan las proteínas de los sistemas ABO y Rh ni antígenos del complejo mayor de histocompatibilidad II (MHC-II); razón por la que llaman la atención como posibles herramientas terapéuticas frente a diversas condiciones patológicas [18,20].

De igual manera, se puede establecer la semejanza entre los microquimerismos y la terapia celular con células madre, en la que se realiza una prediferenciación in vitro de las células con el fin de que se especialicen, proliferen y lleven a cabo las funciones del tejido. Este proceso de prediferenciación in vitro se puede realizar a partir de varias fuentes: a) células madre embrionarias pluripotenciales, b) células madre endógenas, a partir de las cuales se obtienen células madre pluripotenciales inducidas (iPS; del inglés induced Pluripotent Stem), y c) células madre de cordón umbilical, las cuales están compuestas tanto por células madre endógenas como por microquimerismos $[12,13]$.

Los estudios comparativos de las diversas fuentes de prediferenciación in vitro no han sido contundentes frente a las ventajas y desventajas que supone la utilización de cada una de éstas. Algunas investigaciones han encontrado una relación negativa entre el número de pases de las células madre pluripotenciales inducidas y el número de variaciones en el número de copias en los genes (CNV; del inglés, Copy Number Variation) [2I]. Incluso se han encontrado en estas células duplicaciones en los oncogenes tras el cultivo por tiempo prolongado y deleciones en regiones del ADN codificantes para genes supresores de tumores asociadas al proceso de reprogramación a un estado pluripotente. Igualmente, diversos estudios han asociado el proceso de reprogramación epigenética en las células madre pluripotenciales inducidas a la presencia de regiones con metilación diferencial (DMR; del inglés, Differentially Methylated Regions), las cuales se han detectado en genes importantes en el desarrollo celular [13].

En cuanto a la utilización de las células madre embrionarias para terapia celular y otras aplicaciones, existe un debate ético, moral y legal de alcance global; razón por la cual está prohibido o bastante restringido su uso en varios países [22]. El debate moral actual gira en torno a la selección arbitraria de embriones para su manipulación, que implica una importante paradoja: mientras se impide el desarrollo de unas vidas, puede que tal interrupción permita la continuación de otras que se encuentran en estados más avanzados del desarrollo; la cuestión entonces es ¡cuál(es) vale(n) más? [23]. Pasando a aspectos más técnicos, al igual que las células madre pluripotenciales inducidas, las células madre embrionarias han mostrado ser tumorigénicas no solo en modelos múridos, sino también en estudios con primates y en ensayos clínicos en humanos $[24,25]$. 
Por su parte, las células madre de cordón umbilical, sitio de donde se obtienen los microquimerismos, han sido ampliamente utilizadas en diversos estudios, en los que no se han relacionado con la formación de tumores [14,26], incluso, estudios comparativos entre estas células madre y las de médula ósea, han demostrado una mayor tasa de neurogénesis y migración vía paracrina de las células de cordón umbilical, así como un mayor efecto neuroprotector y antinflamatorio, y una menor tasa de apoptosis en los cultivos carentes de oxigeno [26].

\section{Localización cerebral y vía de acceso de los microquimerismos}

Los microquimerismos en el sistema nervioso central se han encontrado localizados con mayor prevalencia en la médula oblonga y, en menor proporción, en el hipocampo, en el resto del lóbulo temporal y en el lóbulo parietal, sin reportes de microquimerismos en el lóbulo frontal ni en la amígdala [I I].

Durante el embarazo, para ajustar el creciente volumen plasmático, se produce una disminución de la resistencia vascular y un aumento de la permeabilidad en todo el tejido vascular incluyendo la barrera hematoencefálica, que en consecuencia se cree que facilita el ingreso de los microquimerismos al cerebro [15]. Como soporte experimental, en modelos múridos se marcaron los microquimerismos con proteína verde fluorescente (GFP; del inglés, Green Fluorescent Protein) y se evaluó posteriormente su localización, encontrándolos ubicados a nivel intracerebral, lo que sugiere que efectivamente pueden cruzar la barrera hematoencefálica [ 16].

Además, la región posterior del piso del IV ventrículo y el hipotálamo son lugares en los que se cree que los receptores neuronales tienen contacto directo con el resto del plasma, lo cual aumenta la permeabilidad cerebral en estas zonas y, posiblemente, da paso a los microquimerismos $[27,28]$. Pese a lo anterior, cabe anotar que aún no existe una explicación clara sobre la manera en que los microquimerismos cruzan la barrera hematoencefálica pues las existentes se fundamentan en modelos teóricos.

\section{Complejo mayor de histocompatibilidad y microquimerismos}

Las implicaciones de los microquimerismos en la salud del organismo hospedero son motivo de debate pues los estudios han arrojado resultados variables $y$, en ocasiones, contradictorios frente al papel que desempeñan como promotores de bienestar o de enfermedad $[9,10]$. Las consecuencias de los microquimerismos dependen de diversos factores como su origen (p. ej. maternal o fetal), el tipo de células que son traspasadas, el tiempo transcurrido desde la adquisición del quimerismo y la edad del receptor $[9,10,29,30]$. Todos estos elementos se correlacionan ampliamente con las proteínas del sistema de antígenos leucocitarios humanos (HLA; del inglés, Human Leukocyte Antigen) [9,10,31].

Los antígenos leucocitarios humanos (HLA) son proteínas de la superficie de la membrana celular encargadas de mediar la aceptación o rechazo de un injerto por las células del sistema inmune mediante un mecanismo de identificación de las moléculas propias y diferenciación de 
las extrañas, siendo estas últimas las responsables de desencadenar una respuesta inmunológica de rechazo. Debido al alto polimorfismo genético del complejo mayor de histocompatibilidad (MHC; del inglés, Major Histocompatibility Complex) existen múltiples combinaciones de estas moléculas. Por consiguiente, sólo dos individuos que presenten los mismos o similares determinantes antigénicos de las moléculas de HLA serán inmunológicamente compatibles y el intercambio de sus células se espera que no genere una respuesta de rechazo [32,33].

La activación de la respuesta inmune de rechazo frente a un agente externo (p. ej. el injerto) se da por medio de la presentación de aloantígenos a los linfocitos T del receptor. Éstos reconocen por medio de un receptor específico, el TCR (del inglés, T cell receptor), moléculas intactas (sin procesar) del complejo mayor de histocompatibilidad del injerto (presentación directa) o las porciones antigénicas procesadas de estas moléculas y otros aloantígenos, unidos de manera no covalente a las moléculas del HLA del receptor (presentación indirecta) de la misma manera que se realiza la presentación antigénica de cualquier proteína extraña (p. ej. microbiana), generando en ambos casos tanto linfocitos T citotóxicos (CD8+) como ayudadores (CD4+) que reconocen los antígenos del injerto y contribuyen a la respuesta inmune de rechazo [34].

En la respuesta inmune adquirida hay, principalmente, dos tipos de linfocitos y dos clases de moléculas HLA; la estimulación de uno u otro tipo de linfocitos depende del sitio de adquisición del antígeno y del tipo de molécula HLA al que éste encuentre asociado. Los antígenos intracelulares son presentados por las moléculas HLA de clase I, presentes en todas las células nucleadas del organismo, y estimulan la acción de los linfocitos T CD8+ para que destruyan las células nucleadas en el injerto que expresan los aloantígenos, mientras que los antígenos extracelulares adquiridos por la vía vesicular endosomal (p. ej. por fagocitosis), son presentados por las moléculas HLA de clase II, presente sólo en células especializadas (células presentadoras de antígenos, p. ej. monocitos, macrófagos, células dendríticas y linfocitos B), estimulan a los linfocitos T CD4+ para producir citoquinas inflamatorias que inducen el daño de los injertos [29,32,34].

\section{Respuesta inmune asociada a microquimerismos}

El sistema HLA en la evaluación de los microquimerismos fetomaternales adquiere relevancia en la medida en que los microquimerismos actúan como injertos en el receptor al ser células provenientes de otro organismo $y$, por tanto, pueden desencadenar una respuesta inmune mediada por los antígenos HLA. No obstante, esta respuesta no necesariamente se da y, en caso de que se presente, es un fenómeno que ocurre con grados de intensidad variables que dependen de diversos factores como las diferencias entre los haplotipos (entendidos como bloques de genes del sistema HLA) de la madre y el feto [9]. De esta manera, el microquimerismo se considera un potencial desencadenante de la respuesta inmune, pero con la posibilidad de que si la madre y el feto comparten cierto número de alelos esta activación es baja o nula [3।]. Además, se considera que el microquimerismo fetomaternal puede resultar dañino o beneficioso dependiendo de la compatibilidad entre el sistema HLA fetal y el maternal [9,31,35].

En el caso de la artritis reumatoide, una enfermedad autoinmune en la que se han identificado alelos de protección y de riesgo del sistema HLA, algunos estudios han demostrado que si el microquimerismo, independientemente de su origen, contiene la variable de protección y el hospedero porta la variable de riesgo, las células nuevas aportan un decremento en la respuesta 
autoinmune, pero, en caso contrario, el microquimerismo constituye un factor de riesgo no sólo frente a la artritis reumatoide sino también frente a todas las demás enfermedades de origen autoinmune $[9,10,36]$.

Por su parte, estudios sobre microquimerismos adquiridos en el embarazo en mujeres con esclerosis sistémica, un trastorno autoinmune del tejido conectivo, han demostrado que si el hospedero y el injerto son homólogos en sus alelos del complejo HLA la expresión de la enfermedad aumenta, lo que sugiere una sobrexpresión de la respuesta inmune [10]. Sin embargo, estudios de biopsias de piel de mujeres que padecen la enfermedad, analizadas por medio de las técnicas de hibridación fluorescente in situ (FISH; del inglés, Fluorescence In Situ Hybridization) y reacción en cadena de la polimerasa (PCR; del inglés polymerase chain reaction), revelaron una mayor cantidad de células quiméricas en las regiones sanas respecto a las regiones afectadas, lo que indica un papel del microquimerismo en la reparación más que como un factor causal de la enfermedad [9,37]. Otro ejemplo de microquimerismo como agente protector condicionado se ha descrito en los trasplantes de riñón entre hermanos, en los que se produce una mayor tolerancia al trasplante si el donante es portador del alelo materno que el receptor tiene únicamente en las células quiméricas maternas que adquirió durante su desarrollo fetal [9].

Pese a que los mecanismos de acción de los microquimerismos aún no son claros, se ha postulado que el tipo de respuesta que se da en el hospedero frente a los microquimerismos depende de la proporción de células T reguladoras en el hospedero respecto a la proporción de células $T$ efectoras adquiridas, dado que las $T$ reguladoras silencian la respuesta frente al injerto generando tolerancia y las T efectoras inducen sensibilización frente al sistema HLA del injerto $[9,19]$. De forma experimental se ha demostrado que existe un mayor número de microquimerismo materno si la madre es compatible con el hijo en el gen HLA-DQBI, ambos comparten exactamente los mismos alelos HLA o si ésta es homocigótica para un alelo compartido con el feto; hallazgos que son consistentes con los resultados obtenidos en los modelos con ratones. En cuanto a los microquimerismos fetales se ha demostrado que el genotipo de moléculas HLA clase II que se encuentra más frecuentemente asociado con persistencia de estas células fetales en la madre es el HLA-DQA $|* 050|$ [|0,| I,3।].

Desde el punto de vista inmunológico, la incorporación en el organismo de una célula madura es diferente que la de una célula en los estadios previos de diferenciación, ya que, por ejemplo, las células $T$ vírgenes del sistema inmunológico fetal tienen una mayor tendencia a diferenciarse en células $T$ reguladoras respecto a las células $T$ vírgenes del adulto [9] y se encargan de la inmunotolerancia en el feto frente a antígenos de la madre y persisten en el individuo hasta su adultez. Uno de los mecanismos que explica este fenómeno sugiere que las células maduras de la madre atraviesan la placenta y residen en los nódulos linfáticos fetales, donde inducen el desarrollo de las células T reguladoras [38].

Por otra parte, se han propuesto dos mecanismos para explicar la tolerancia de la madre frente a los microquimerismos fetales: a) la maduración de las células $T$ fetales en el timo materno, donde aquellas aloreactivas contra antígenos maternos pueden ser eliminadas y aquellas con cierto nivel de histocompatibilidad pueden ser seleccionadas positivamente; o b) la diferenciación de las células $T$ fetales antígeno específicas hacia un fenotipo regulador (células T reguladoras), que no atacan las células maternas y luego pueden migrar a la madre y probablemente influenciar la función de las células del sistema inmune materno [9,38]. 
No obstante, aún no está claro el efecto acumulativo de los microquimerismos, es decir, si existe competencia entre los distintos injertos que se pueden adquirir a lo largo de la vida, ya sea que provengan de varios hijos, hermanos, transfusiones de sangre o trasplantes. Un estudio realizado por Gammill y colaboradores en el 2010 [39] en 138 mujeres sanas en edad reproductiva, demostraron que el aumento en la paridad (número de partos) está fuertemente asociado con la disminución de los microquimerismos maternos. Aunque no encuentran una relación entre la paridad y los microquimerismos fetales, los autores sugieren que la ausencia de un aumento en los microquimerismos fetales puede representar la competencia entre los injertos, con el predominio de una única fuente, lo que conduce a que la prevalencia global de cualquier microquimerismo fetal no se altere. Entretanto, señalan que el decremento en los microquimerismos maternos con el aumento de la paridad podría representar el reemplazo de los microquimerismos maternos por los microquimerismos fetales. Estos hallazgos combinados sugieren que la adición de los injertos a un hospedero no resulta en un número aditivo de injertos detectables, pero sí a la interacción dinámica o competitiva de ambos injertos, los microquimerismos fetales y los maternos.

El microquimerismo, especialmente de hijo a madre, ha sido postulado como un factor de protección frente a diversas enfermedades, pues supone un aumento en el repertorio inmunológico de la madre que le provee nuevos mecanismos de defensa [30]. Incluso, se ha visto implicado en la disminución de la incidencia de diversos tipos de cáncer, razón por la que se ha propuesto cierto rol en la reparación de tejidos, la proliferación celular y la vigilancia inmunológica $[40,4 \mathrm{I}]$. Además, como ya se describió, su presencia en las regiones sanas de la piel de mujeres que padecen esclerosis sistémica ha permitido postular que el microquimerismo tiene una función en la regeneración del tejido. Para que este proceso se dé, se deben cumplir dos requisitos: a) el microquimerismo debe migrar a un área en la cual haya lesión y b) el microquimerismo debe tener capacidad de diferenciación [10].

Por su parte, el efecto nocivo del microquimerismo dependerá de una gran diversidad de factores entre los cuales se han identificado el microambiente celular al que migra, las características genéticas de las células del organismo hospedero y de la quimera, el grado de diferenciación y el lugar de localización, entre otros. Por tal razón, su clasificación como nocivo o benéfico es multifactorial $[9,10,26,38]$.

\section{Enfermedades neurodegenerativas}

\section{Enfermedad de Alzheimer}

La enfermedad de Alzheimer es un tipo de demencia crónica y global que presenta una sintomatología clínica e histopatológica característica; clínicamente se presenta como un proceso progresivo de deterioro cognitivo y funcional de inicio insidioso al que en el transcurso del tiempo se asocian cambios en el estado de ánimo con alteraciones psicóticas y de la conducta $[2-4,42,43]$ y, neuropatológicamente se encuentran alteraciones que se sitúan principalmente en áreas temporo-parietales de la corteza cerebral [42,44]. El diagnóstico de enfermedad de Alzheimer se estable cuando el paciente presentan las siguientes condiciones: a) deterioro de la memoria y de otro proceso cognitivo, b) un curso progresivo del deterioro y c) ausencia de 
otra etiología, es decir, que se debe establecer el diagnóstico diferencial con otras demencias $[42,43]$.

Las alteraciones propias de la enfermedad de Alzheimer se caracterizan por agregados extracelulares de 39 a 42 repeticiones de la proteína $\beta$-amiloide y degeneración neurofibrilar intracelular por fosforilación excesiva de la proteína tau $(\tau)$. Estas alteraciones son neurotóxicas y se cree que son responsables de las alteraciones sinápticas, la pérdida neuronal, la degeneración del sistema colinérgico y atrofia cerebral $[42,44]$. Además, se han identificado diversos factores de predisposición genética para sufrir Alzheimer, entre los cuales las alteraciones más importantes han sido identificadas en los genes que codifican para la presenilina- I, presenilina-2 y la proteína precursora amiloide y en el alelo ApoE4 de la apoliproteína E [45].

Teniendo en cuenta la relación entre el Alzheimer y los microquimerismos, diversos estudios han demostrado una relación positiva entre la enfermedad y el embarazo, apuntando a que un mayor número de embarazos lleva a una presentación más temprana de los síntomas propios de esta demencia [46,47]; sin embargo, estos estudios dejan a un lado las variables de susceptibilidad genética. En investigaciones realizadas en mujeres con el alelo de riesgo para el gen de la apoliproteína E (APOE 4) no se ha establecido una asociación entre la frecuencia de éste y la paridad [48]; además, parece que la paridad no afecta la edad de inicio de los síntomas de Alzheimer en mujeres con dicho alelo. Entretanto, en el envejecimiento normal se ha encontrado una relación inversa entre el número de embarazos y el deterioro cognitivo, lo que sugiere un papel protector del embarazo sobre la capacidad cognitiva [47].

\section{Respuesta inflamatoria en la enfermedad de Alzheimer}

En la enfermedad de Alzheimer se presenta un proceso inflamatorio caracterizado por neurotoxicidad, gliosis reactiva y estrés oxidativo [42] en la que los microquimerismos podrían desempeñar un papel relevante debido a su implicación en la modulación de la respuesta inmune [3I,42].

Entre las células reguladoras y efectoras de la respuesta inmune en el sistema nervioso central se encuentran los linfocitos T y las células de la glía, las cuales desempeñan ambos papeles. Las células de la glía se clasifican en microglía, astrocitos y oligodendrocitos, cada una con características y funciones específicas que se han relacionado con la enfermedad de Alzheimer, las cuales se describen a continuación [29,49-5I]:

$\rightarrow$ Microglía: su actividad se ha relacionado con la vigilancia inmunológica, la expresión de mediadores y receptores pro y antinflamatorios, el ataque y la remoción de patógenos, y la secreción de factores de regeneración del tejido. La sobrestimulación de estas células induce neurotoxicidad, excitotoxicidad [49-52] y aumento en la secreción de factores inflamatorios relacionados con la neurodegeneración. Estas células se han encontrado en las regiones histopatológicamente vulnerables de la enfermedad de Alzheimer. Además, aunque los depósitos de $\beta$-amiloide activan la respuesta inflamatoria del cerebro, la microglía no es capaz de fagocitar tales depósitos, lo que conduce a daño en las neuronas adyacentes [49].

$\rightarrow$ Astrocitos: entre sus funciones se encuentran la secreción y el reciclaje de neurotransmisores, la homeostasis iónica, la regulación del metabolismo energético, la remodelación de la sinapsis, la modulación del estrés oxidativo, la presentación antigénica y la excitotoxicidad 
[52]. En la enfermedad de Alzheimer los astrocitos rodean las placas $\beta$-amiloide aislando las células sanas de las patógenas [50]. Algunos estudios sugieren que los astrocitos tienen función de degradación de las placas, lo que genera la liberación de moléculas neurotóxicas promotoras de la degeneración neuronal [49]. La exposición de los astrocitos a las placas neuríticas ha sido asociada con una reactividad excesiva, lo cual lleva a una degeneración neuronal por sobrexpresión de factores inflamatorios [49-5I].

$\rightarrow$ Oligodendrocitos: son las células que producen las vainas de mielina necesarias para la velocidad del impulso nervioso, por lo cual desempeñan un papel crucial en las sinapsis $[49,50]$. Algunos estudios por imaginología y en modelos de ratones transgénicos han reportado lesiones y alteraciones desmielinizantes en la enfermedad de Alzheimer, relacionadas con la presencia de depósitos del $\beta$-amiloide, los cuales resultan tóxicos para este tipo de células [5।,52].

Por otra parte, las neuronas tienen como función la expresión de moléculas protectoras contra un ataque inflamatorio. En la enfermedad de Alzheimer se ha encontrado deficiencia en estos mecanismos de defensa en regiones patológicamente vulnerables, tales como la corteza y el hipocampo [5I].

Además, el sistema del complemento, una vía inflamatoria crucial en la ejecución de respuestas inmunológicas y cuya función es el reconocimiento de patógenos y patrones moleculares asociados a tejidos dañados y células que están muriendo [52], se encuentra activado en la enfermedad de Alzheimer, donde ejerce funciones reguladoras y colaborativas en la eliminación de las células en proceso de degeneración y de agregados proteicos. Sin embargo, la activación de este sistema también puede generar una inflamación innecesaria y dañina, la cual ha sido asociada a ciertos factores genéticos. Las citoquinas y quimioquinas también desempeñan un papel en la activación del sistema inmune y se expresan anormalmente en el cerebro de personas con enfermedad de Alzheimer, activando las células de la glía y generando inflamación [49,52].

Por último, a partir de diversos hallazgos en genómica molecular se explica que la expresión de los genes asociados a la inflamación aumentan en la vejez y que precisamente el aumento de esta expresión genética está acentuado en la enfermedad de Alzheimer [49]. De acuerdo con lo anterior, se ha observado que a medida que aumenta la edad se aumentan los transcriptos relacionados con el complejo mayor de histocompatibilidad, el sistema del complemento y la fagocitosis en personas con cognición normal en comparación con aquellos que atraviesan un proceso demencial, lo que sugiere que un aumento en la inmunidad cerebral puede ser protector y que la incapacidad para adaptarse al proceso de envejecimiento es un factor de riesgo contra enfermedades neurodegenerativas [5 I].

\section{Demencia frontotemporal lobular}

Este tipo de demencia se caracteriza por una degeneración frontotemporal lobular, en la cual se observa una atrofia progresiva de los lóbulos frontales y temporales bilaterales asociada a pérdida neuronal y gliosis [42,53]. Por lo general, esta condición patológica se presenta sin coexistencia con la enfermedad de Alzheimer [42]. Los principales signos clínicos de esta enfermedad incluyen alteraciones de la conducta social, el lenguaje y la memoria; sin embargo, su presentación es bastante heterogénea $[42,53]$. 
Existen diversas variantes de la demencia frontotemporal lobular, cuyas manifestaciones clínicas e histopatológicas difieren ampliamente entre sí. Desde una perspectiva clínica se clasifican según el orden de aparición de los síntomas o de acuerdo con el síntoma preponderante, encontrando tres subtipos principales: a) la variante frontal, en la cual se puede presentar un síndrome disejecutivo, desinhibición comportamental o apatía dependiendo de la región que se vea afectada, b) la demencia semántica, en la cual se pierde el significado del lenguaje en general, y c) la afasia progresiva no fluente, en la que se afecta la producción del lenguaje $[42,44,53,54]$.

Histopatológicamente las distintas formas de la demencia frontotemporal lobular se clasifican según el tipo de inclusión en el citoplasma neuronal como: a) taupatías, en las cuales hay una agregación de la proteína tau ligada a microtúbulos, y b) ubiquinopatías, en las que hay agregación de la ubiquitinas [42,54-56]. En el 40\% de las personas que padecen demencia frontotemporal lobular existe una historia familiar de demencia y en el $10 \%$ su forma de herencia es autosómica dominante $[6,53]$. Dentro de los genes que han sido asociados a esta enfermedad, el gen de la proteína tau asociada a los microtúbulos (MAPT) y el gen de la progranulina (GRT) dan cuenta de alrededor del $60 \%$ de los casos de demencia frontotemporal lobular familiar, y el gen del cromosoma 9 marco de lectura abierta 72 (C9ORF72; del inglés, Chromosome 9 Open Reading Frame 72) aparece implicado en alrededor del I2\% de los casos [53,56].

\section{Respuesta inflamatoria}

\section{en la demencia frontotemporal lobular}

La demencia frontotemporal lobular también se ha relacionado con la aparición de una respuesta inflamatoria, por lo que los mecanismos de actuación de los microquimerismos podrían relacionarse con esta alteración a través de la modulación del sistema inmune. Estudios recientes en ratones knock-out para la progranulina han sugerido una relación entre la deficiencia de esta proteína y los patrones comportamentales similares a los de la degeneración frontotemporal lobular, en la que se observan alteraciones en la conducta social con preservación de la memoria hasta etapas tardías del desarrollo de la enfermedad, acompañada de microgliosis y astrogliosis [57]. Esto sugiere que la sobreactivación de las células de la glía y la inflamación neuronal que resultan de la deficiencia de la progranulina podrían mediar la disfunción neuronal de pacientes con demencia frontotemporal lobular [57,58].

Dado que las alteraciones en la demencia frontotemporal lobular relacionadas con taupatías y ubiquitinopatías se deben a inclusiones intracelulares, es factible que los mecanismos de acción de la respuesta inflamatoria en esta enfermedad sean similares a los de la enfermedad de Alzheimer $[42,57,58]$. De esta manera, al relacionar los resultados obtenidos en la enfermedad de Alzheimer con las inclusiones de la proteína tau y de ubiquitina observadas en la demencia frontotemporal lobular, los microquimerismos son potencialmente protectores frente a estas condiciones patológicas.

\section{Técnicas para la identificación de microquimerismos}

Para la detección de microquimerismos las técnicas que más se han utilizado son la reacción en cadena de la polimerasa cuantitativa en tiempo real y la hibridación fluorescente in situ (FISH) $[9,16,59]$. La reacción en cadena de la polimerasa cuantitativa ha sido mayormente utilizada 
para amplificar el gen DYSI4 ubicado en el cromosoma Y, que identifica células masculinas en cerebros femeninos, que se asumen como microquimerismos debido a su baja cantidad $[11,37,60,61]$.

La hibridación in-situ fluorescente es más utilizada para identificar células femeninas $(X X)$ en un hombre o masculinas (XY) en una mujer (mismatch), ya que el grado de fluorescencia varía diferencialmente según la carga genética, lo cual hace posible diferenciar las células masculinas y femeninas [9]. Otros autores proponen la utilización de esta técnica, pero no atada a la identificación del sexo sino a la determinación de polimorfismos mediante la detección de una subclase de número de copias, llamada variante de delección. Esta técnica fue denominada por sus autores «Polymorfic Deletion Probes (PDP)», la cual detecta el número de nucleótidos en una secuencia de ADN [62].

Para identificar los alelos del complejo mayor de histocompatibilidad existen diversos métodos que se basan en la unión diferencial de péptidos a estas moléculas. Debido al alto polimorfismo que presenta el complejo mayor de histocompatibilidad, que da lugar a múltiples moléculas cada una con un potencial de unión a un conjunto único de péptidos, los posibles resultados de unión a estos alelos se encuentran disponibles en bases de datos. Un ejemplo claro de esto es que para 201 I ya se habían identificado que menos de 30 alelos de los 1.457 que componen el sistema HLA-II se unían diferencialmente en términos cuantitativos a más de 200 proteínas [63,64].

La detección de los microquimerismos en conjunto con la historia clínica del paciente y la caracterización histopatológica del tejido cerebral se considera que hará posible la identificación de la implicación, si es que la hay, de los microquimerismos en las enfermedades neurodegenerativas. Los hallazgos experimentales de los microquimerismos realizados hasta la fecha, así como los avances en la terapia celular con células madre, con la cual guardan ciertas similitudes, indican un posible poder protector y curativo de estos pequeños depósitos de células de un organismo extraño dentro de otro. En la medida en que se avance hacia el tratamiento de las enfermedades neurodegenerativas se estará aportando a la solución de un problema inminente con tendencia a aumentar.

\section{Conclusiones}

El estudio de la presencia de microquimerismos en enfermedades neurodegenerativas ha sido muy limitado y se ha reducido a la enfermedad de Alzheimer y la esclerosis múltiple. Además, gran parte de las investigaciones se han realizado en modelos múridos, lo que hace aún más remota la comprensión actual de la función que ejercen sobre este tipo de enfermedades. En general, se ha encontrado que estos pequeños depósitos de células en un organismo provenientes de otros tienen un papel benéfico sobre las enfermedades neurodegenerativas [9-। I].

A partir de un modelo múrido en el cual se cruzaron ratones silvestres con ratones transgénicos marcados con proteína verde fluorescente se observó que los microquimerismos migran a las mismas localizaciones que las células de la glía y las neuronas y que adquieren su morfología y marcadores inmunocitoquímicos, lo cual es indicativo de una especialización hacia las mismas [16].

De acuerdo con lo anterior, otros estudios con ratones han observado que el microquimerismo tiene la capacidad de atravesar la barrera hematoencefálica y se ha propuesto que presentan el 
proceso de diferenciación celular propio de la neurogénesis adulta. Estos modelos sugieren que el microquimerismo actúa como un agente regenerador de tejido en el ataque cerebral. Además, al inyectar células de cordón umbilical humano a ratones con esta dolencia disminuyeron los síntomas comportamentales asociados al mismo. No obstante, a pesar de estas evidencias, en algunos estudios no se han hallado microquimerismos en el tejido cerebral, razón por la cual surge la cuestión sobre la cantidad, la prevalencia y la permanencia de éstos en el organismo hospedero; elementos que inciden sobre el impacto que pueden tener sobre la salud [59].

En primates y en roedores se ha propuesto la neurogénesis en dos regiones del cerebro adulto: a) la región subventricular de los ventrículos laterales, de donde las células progenitoras migran hacia el bulbo olfatorio, luegar en el que se diferencian a neuronas y b) el giro dentado hipocampal, de donde migran hacia la región granulosa para diferenciarse en células granulosas. Es relevante el hecho de que las células granulosas se encuentren en el hipocampo, más específicamente en el giro dentado, regiones ampliamente relacionadas con la enfermedad de Alzheimer, ya que efectivamente los microquimerismos pueden actuar en el cerebro a través de esta vía [17].

Cabe recordar que los microquimerismos pueden ser benéficos o deletéreos en enfermedades relacionadas con respuestas inflamatorias dependiendo de sus características $[8,15,31,36]$, lo que hace de gran importancia la realización de estudios sobre la carga genética de los microquimerismos y su papel en la salud y la enfermedad del organismo hospedero. Teniendo en cuenta que los microquimerismos de origen fetal comparten el 50\% del genoma con el organismo receptor, es decir, la madre, y que el otro 50\% proviene del genoma del padre, es posible que las variables genéticas foráneas que sean introducidas tengan tanto alelos de protección como de susceptibilidad, lo que hace que haya microquimerismos que promuevan la salud y otros que, por el contrario, promuevan la degeneración de los tejidos en la madre.

Sin embargo, se ha encontrado una relación inversa entre la cantidad de microquimerismos y la enfermedad de Alzheimer, pues de acuerdo con los estudios que se han realizado en humanos, ha sido estadísticamente significativa la diferencia en la cantidad de microquimerismos en cerebros de mujeres que padecieron la enfermedad frente a aquellas que no la padecieron, siendo mayor en las segundas $[11,17]$.

\section{Agradecimientos}

Este trabajo de revisión fue realizado gracias a los aportes del Comité para el Desarrollo de la Investigación (CODI) y la Universidad de Antioquia mediante la financiación del proyecto de investigación «Evaluación de microquimerismos en enfermedades neurodegenerativas», bajo el código 2558.

\section{Bibliografía}

I. Lindvall O, Kokaia Z. Stem cells in human neurodegenerative disorders--time for clinical translation? J Clin Invest 2010; 120: 29-40.

2. Alzheimer's Association. 2013 Alzheimer's disease facts and figures. Alzheimers Dement 20।3; 9: 208-245.

3. Modig K, Drefahl S, Ahlbom A. Limitless longevity: comment on the Contribution of rectangularization to the secular increase of life expectancy. Int J Epidemiol 2013; 42: 914-916.

4. Reitz C, Mayeux R. Alzheimer disease: epidemiology, diagnostic criteria, risk factors and biomarkers. Biochem Pharmacol 2014; 88: 640-65।. 
5. Camps Calzadilla E, Gámez Fonseca M, Borroto M, Prado Martínez C. Evaluación nutricional de los centenarios cubanos y su relación con biomarcadores del envejecimiento. Rev Cubana Invest Bioméd 2013; 32: 57-64.

6. Onyike CU, Diehl-Schmid J. The epidemiology of frontotemporal dementia. Int Rev Psychiatry 2013; 25: I30137.

7. Galimberti D, Dell'Osso B, Altamura AC, Scarpini E. Psychiatric Symptoms in Frontotemporal Dementia: Epidemiology, Phenotypes, and Differential Diagnosis. Bio Psychiatry 20 15; Abr 8 [Epub ahead of print].

8. Boddy AM, Fortunato A, Wilson Sayres M, Aktipis A Fetal microchimerism and maternal health: a review and evolutionary analysis of cooperation and conflict beyond the womb. Bioessays 2015; Ago 28 [Epub ahead of print].

9. Nelson JL. The otherness of self: microchimerism in health and disease. Trends Immunol 20 I2; 33: 42I-427.

10. Boyon C, Collinet P, Boulanger L, Rubod C, Lucot JP, Vinatier D. Fetal microchimerism: benevolence or malevolence for the mother? Eur J Obstet Gynecol Reprod Biol 201 I; I58: |48-152.

II. Chan WF, Gurnot C, Montine TJ, Sonnen JA, Guthrie KA, Nelson JL. Male microchimerism in the human female brain. PLoS One 2012; 7: e45592.

12. Kim SU, Lee HJ, Kim YB. Neural stem cell-based treatment for neurodegenerative diseases. Neuropathology 2013; 33: 49|-504.

13. Robinton DA, Daley GQ. The promise of induced pluripotent stem cells in research and therapy. Nature 20।2; 48I: 295-305.

14. Hsieh JY, Wang HW, Chang SJ, Liao KH, Lee IH, Lin WS, et al. Mesenchymal stem cells from human umbilical cord express preferentially secreted factors related to neuroprotection, neurogenesis, and angiogenesis. PLoS One 20।3; 8: e72604.

15. Dawe GS, Tan XW, Xiao ZC. Cell migration from baby to mother. Cell Adh Migr 2007; I: 19-27.

16. Tan XW, Liao H, Sun L, Okabe M, Xiao ZC, Dawe GS. Fetal microchimerism in the maternal mouse brain: a novel population of fetal progenitor or stem cells able to cross the blood-brain barrier? Stem Cells 2005; 23 | 443- 452.

17. Mezey E, Brownstein MJ. Do circulating cells transdifferentiate and replenish stem cell pools in the brain and periphery? Bioessays 20I5; 37: 398-402

18. Velázquez FN, Caputto BL. Aspectos generales de las células madre y su potencial aplicación en la medicina regenerativa. Bitácora Digital 2013; I: I-6.

19. Khosrotehrani K, Leduc M, Bachy V, Nguyen Huu S, Oster M, Abbas A, et al. Pregnancy allows the transfer and differentiation of fetal lymphoid progenitors into functional T and B cells in mothers. I Immunol 2008; I 80: 889-897.

20. Bongso A, Fong CY. The therapeutic potential, challen- ges and future clinical directions of stem cells from the Wharton's jelly of the human umbilical cord. Stem Cell Rev 2013; 9: 226-240.

21. Hussein SM, Batada NN, Vuoristo S, Ching RW, Autio $\mathrm{R}$, Narva E, et al. Copy number variation and selection during reprogramming to pluripotency. Nature 2011; 47l: 58-62.

22. King NM, Perrin J. Ethical issues in stem cell research and therapy. Stem Cell Res Ther 20। 4; 5: 85.

23. Andorno R. La dimensión biológica de la personalidad humana: El debate sobre el estatuto del embrión. Cuad Bioét 2004; 1: 29-36.

24. Lee AS, Tang C, Rao MS, Weissman IL, Wu JC. Tumorigenicity as a clinical hurdle for pluripotent stem cell therapies. Nat Med 20 I3; 19: 998-1004

25. Kuroda T, Yasuda S, Sato Y. Tumorigenicity Studies for Human Pluripotent Stem Cell-Derived Products. Biol Pharm Bull 2013; 36: 189-192.

26. Jin HJ, Bae YK, Kim M, Kwon SJ, Jeon HB, Choi SJ, et al. Comparative analysis of human mesenchymal stem cells from bone marrow, adipose tissue, and umbilical cord blood as sources of cell therapy. Int J Mol Sci 20I3; |4: |7986-| 800 |

27. Snell RS. Sistema ventricular, el líquido cefalorraquídeo y barreras hematoencefálicas y hematorraquídea. In: Snell RS ed. Neuroanatomía Clínica (ed 5a). Buenos Aires, Argentina: Editorial Médica Panamericana; 2003: $443-$ 472

28. Mezey É, Key S, Vogelsang G, Szalayova I, Lange GD Crain B. Transplanted bone marrow generates new neurons in human brains. Proc Natl Acad Sci USA 2003, 100: 1364-1369.

29. Sawcer S, Hellenthal G, Pirinen M, Spencer CC, Patsopoulos NA, Moutsianas L, et al. Genetic risk and a primary role for cell-mediated immune mechanisms in multiple sclerosis. Nature 201 I; 476: 214-219.

30. Nijagal A, Wegorzewska M, Jarvis E, Le T, Tang $\mathrm{Q}$, MacKenzie TC. Maternal T cells limit engraftment after in utero hematopoietic cell transplantation in mice. J Clin Invest 201 I; 121: 582-592.

3I. Leveque L, Khosrotehrani K. Feto-maternal allo-immunity, regulatory $T$ cells and predisposition to auto-immunity. Does it all start in utero? Chimerism 20।4; 5: 59-62.

32. Garavito G, Iglesias A, Egea E, Jaraquemada D, Martínez P, Egea EE. Una aproximación al significado biológico del polimorfismo del Complejo Mayor de Histocompatibilidad. El modelo de la asociación HLA y AR|**. Salud Uninorte Barranquilla (Col) 2002; 16: 53-72

33. Montano-Frías JE, Rocha-Olivares A, Licea-Navarro AF, Morteo-Ortiz E, Segura-García I, Heckel G. Polimorfismo del gen DQB del complejo principal de histocompatibilidad clase II como bio-indicador en tursiones Tursiops truncatus del Golfo de México y Mar Caribe. Veracruz, México: XXXII Reunión Internacional para el Estudio de los Mamíferos Marinos, 2-6 de mayo. Sociedad Mexicana de Mastozoología Marina (SOMEMMA) 2010. 
34. Abbas AK, Lichtman AH, Pillai S. Transplantation Immunology. En: Abbas AK, Lichtman AH, Pillai S, eds. Cellular and Molecular Immunology (ed 7a). Filadelfia, Estados Unidos: Elsevier Saunders; 20 12: 365-388.

35. Lambert NC. Multiple ways human leukocyte antigens (HLA) could influence chimerism. Chimerism 2013; 4: 41.

36. Kekow M, Barleben M, Drynda S, Jakubiczka S, Kekow J, Brune T. Long-term persistence and effects of fetal microchimerisms on disease onset and status in a cohort of women with rheumatoid arthritis and systemic lupus erythematosus. BMC Musculoskelet Disord 2013; 14: I-8.

37. Irie N. Emerging Questions in Materno-Fetal Microchimerism. Reproductive Sys Sexual Disord 201 I; SI: 002.

38. Mold JE, Michaelsson J, Burt TD, Muench MO, Beckerman KP, Busch MP, et al. Maternal alloantigens promote the development of tolerogenic fetal regulatory $T$ cells in utero. Science 2008; 322: I562-1565.

39. Gammill HS, Aydelotte TM, Guthrie KA, Nkwopara EC, Nelson JL. Cellular fetal microchimerism in preeclampsia. Hypertension 2013; 62: 1062-1067.

40. Eun JK, Guthrie KA, Zirpoli G, Gadi VK. In situ breast cancer and microchimerism. Sci Rep 2013; 3: 2192.

41. Cirello V, Colombo C, Perrino M, De Leo S, Muzza M, Maffini MA, et al. Fetal cell microchimerism in papillary thyroid cancer: A role in the outcome of the disease. Int J Cancer 2015; 137: 2989-2993.

42. Alberca Serrano R, López-Pousa S. Enfermedad de Alzheimer y otras Demencias (ed 4ta). Madrid, España: Editorial Médica Panamericana; 201 I.

43. Morrison J. DSM-5® Guía para el diagnóstico clínico. México D.F., México: Editotial El Manual Moderno; 2015.

44. Kolb B, Whishaw IQ. La memoria. En: Kolb B, Whishaw IQ, eds. Neuropsicología humana (ed 5ta). Madrid, España: Editorial Médica Panamericana; 2006: 447-482.

45. Ringman JM, Coppola G. New genes and new insights from old genes: update on Alzheimer disease. Continuum (Minneap Minn) 20।3; 19: 358-37l.

46. Colucci M, Cammarata S, Assini A, Croce R, Clerici $\mathrm{F}$, Novello $\mathrm{C}$, et al. The number of pregnancies is a risk factor for Alzheimer's disease. Eur J Neurol 2006; 13 : |374-1377.

47. Shansky RM. Sex Differences in the Central Nervous System. Londres, Reino Unido: Academic Press; 2015.

48. Corbo RM, Ulizzi L, Scacchi R, Martinez-Labarga C, De Stefano GF. Apolipoprotein E polymorphism and fertility: a study in pre-industrial populations. Mol Hum Reprod 2004; 10: 617-620.

49. Wyss-Coray T, Rogers J. Inflammation in Alzheimer disease-a brief review of the basic science and clinical literature. Cold Spring Harb Perspect Med 2012; 2: a006346.

50. Lopategui Cabezas I, Herrera Batista A, Pentón Rol G. Papel de la glía en la enfermedad de Alzheimer. Futuras implicaciones terapéuticas. Neurología 20 14; 29: 305 309.

5I. Vinet J, Weering HR, Heinrich A, Kalin RE, Wegner A, Brouwer $\mathrm{N}$, et al. Neuroprotective function for ramified microglia in hippocampal excitotoxicity. I Neuroinflammation 2012; 9: 27.

52. Graeber MB, Streit WJ. Microglia: biology and pathology. Acta Neuropathol 2010; 1 19: 89-105.

53. Riedl L, Mackenzie IR, Forstl H, Kurz A, Diehl-Schmid J. Frontotemporal lobar degeneration: current perspectives. Neuropsychiatr Dis Treat 20 I4; 10: 297-310.

54. Flores Lázaro JC, Ostrosky-Shejet F, Lozano Gutiérrez A. Batería de Funciones Ejecutivas: Presentación. Rev Neuropsi Neuropsiq Neuroc 2008; 8: I4I - 58.

55. Rascovsky K, Hodges JR, Knopman D, Mendez MF, Kramer JH, Grossman M, et al. Reply: Considering the frontomedian cortex in revised criteria for behavioural variant frontotemporal dementia. Brain 20 12; 135: e2 I4e2I4.

56. Whitwell JL, Weigand SD, Boeve BF, Senjem ML, Gunter JL, Dejesus-Hernandez M, et al. Neuroimaging signatures of frontotemporal dementia genetics: C9ORF72, tau, progranulin and sporadics. Brain 2012; 135: 794-806.

57. Yin F, Banerjee R, Thomas B, Zhou P, Qian L, Jia T, et al. Exaggerated inflammation, impaired host defense, and neuropathology in progranulin-deficient mice. J Exp Med 2010; 207: 1 17-128

58. Filiano AJ, Martens LH, Young AH, Warmus BA, Zhou P, Diaz-Ramirez G, et al. Dissociation of frontotemporal dementia-related deficits and neuroinflammation in progranulin haploinsufficient mice. J Neurosci 2013; 33: 5352-5361.

59. Demirhan O, Cekin N, Tastemir D, Tunc E, Guzel Al, Meral D, et al. Are there fetal stem cells in the maternal brain? Neural Regen Res 2013; 8: 593-598.

60. Kruchen A, Stahl T, Gieseke F, Binder TM, Oezcan Z, Meisel R, et al. Fetomaternal Microchimerism Is Associated with Better Outcome in Haploidentical Hematopoietic Stem Cell Transplantation. Blood 2014; 124: 1242-1242

6I. Lee ES, Bou-Gharios G, Seppanen E, Khosrotehrani K, Fisk NM. Fetal stem cell microchimerism: natural-born healers or killers? Mol Hum Reprod 20 0; I6: 869-878.

62. Wu D, Vu Q, Nguyen A, Stone JR, Stubbs H, Kuhlmann $\mathrm{G}$, et al. In situ genetic analysis of cellular chimerism. Nat Med 2009; 15: 215-219.

63. Nielsen M, Justesen S, Lund $O$, Lundegaard $C$, Buus S. NetMHCllpan-2.0 - Improved pan-specific HLA-DR predictions using a novel concurrent alignment and weight optimization training procedure. Immunome Res 2010; 6: 9 .

64. Zhang L, Udaka K, Mamitsuka H, Zhu S. Toward more accurate pan-specific MHC-peptide binding prediction: a review of current methods and tools. Brief Bioinform 2012; 13: 350-364. 

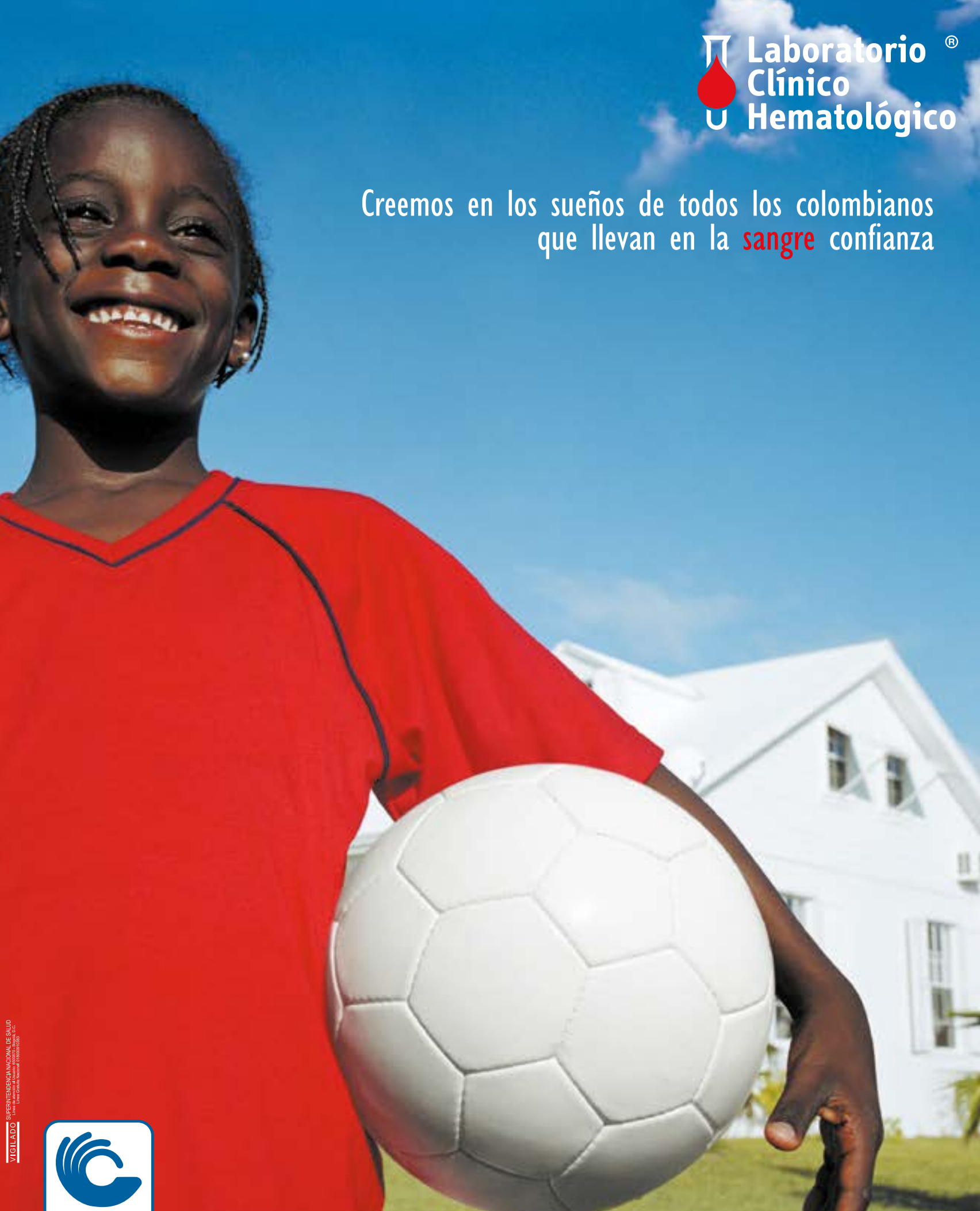

ISO 9001

\$ icontec

Sede Principal Poblado: Carrera 43C No. 5-33

Toma de Muestras: Centro Comercial Sandiego, Torre Norte, Piso 10, No. 1034

Teléfono: 4444200 - www.lch.co - Medellín 